\title{
UNA ESPECIE NUEVA DE MACROCOPTURUS HELLER (COLEOPTERA: CURCULIONIDAE: CONODERINAE) DE GUERRERO, MÉXICO Y DESCRIPCIÓN DE SUS ESTADOS INMADUROS
}

\author{
Raúl MuÑIZ-VÉLEZ† y María Magdalena ORDóÑEZ-RESÉNDIZ1 \\ ${ }^{1}$ Museo de Zoología, Facultad de Estudios Superiores Zaragoza, Universidad Nacional Autónoma \\ de México, Av. Guelatao 66, Ejército de Oriente, 09230 Iztapalapa, D.F., MÉXICO. \\ mor@servidor.unam.mx
}

Muñíz-Vélez, R.† \& Ma. M. Ordóñez-Reséndiz. 2010. Una especie nueva de Macrocopturus Heller (Coleoptera: Curculionidae: Conoderinae) de Guerrero, México y descripción de sus estados inmaduros. Acta Zool. Mex. (n. s.), 26(2): 249-258.

RESUMEN. Se describe una especie nueva de Macrocopturus Heller encontrada en Bursera citronella Mc Vaugh \& Rzedowski en Guerrero, México. M. bruserophagus, nueva especie, es más ancha y plana dorsalmente que otras especies del género, y tiene los fémures carenados y unidentados. Se describen también los estados inmaduros de larva y pupa.

Palabras clave: Macrocopturus, Conoderinae, Curculionidae, Guerrero, Bursera, copal.

Muñíz-Vélez, R.† \& Ma. M. Ordóñez-Reséndiz. 2010. A new species of Macrocopturus Heller (Coleoptera: Curculionidae: Conoderinae) from Guerrero, Mexico, and the description of immature stages. Acta Zool. Mex. (n. s.), 26(2): 249-258

ABSTRACT. A new species of Macrocopturus Heller found in Bursera citronella Mc Vaugh \& Rzedowski in Guerrero, Mexico is described. M. bruserophagus, new species, is proportionally broader and more flattened above than other species of the genus, and has carinate femora armed with ventral teeth. Larvae and pupa are described also.

Key words: Macrocopturus, Conoderinae, Curculionidae, Guerrero, Bursera, copal.

\section{INTRODUCCIÓN}

La subfamilia Conoderinae Schönherr, reconocida como Zygopinae hasta hace poco tiempo (Alonso-Zarazaga \& Lyal 1999), es uno de los grupos más grandes de Curculionidae, incluyendo aproximadamente 216 géneros y 1500 especies (Kojima

\footnotetext{
$†$ El manuscrito original de este documento fue elaborado por el M. en C. Raúl Muñiz Vélez, destacado entomólogo mexicano, especialista en Curculionidae. Al momento de su desafortunado fallecimiento, el 14 de marzo de 2008, se encontraban en proceso las figuras que ilustran este trabajo. Esta publicación es una pequeña fracción de la labor que queda pendiente con el deceso de mi estimado maestro.
}

Recibido: 29/01/2009; aceptado: 04/05/2010. 
\& Lyal 2002). Los adultos se caracterizan por la presencia de un canal prosternal donde se aloja el rostro, grandes ojos que ocupan la mayor parte de la cabeza en algunos grupos y la ausencia de lóbulos postoculares sobre el margen anterolateral del pronoto (Hespenheide 2002). Existe una gran diversidad estructural dentro de la subfamilia, principalmente en las formas neotropicales (Hespenheide 2002), que en su mayoría son poco conocidas.

En el estado de Guerrero se encontró una especie desconocida de Conoderinae durante el desarrollo del proyecto "Taxonomía y prospección del hábitat de las poblaciones de Bursera secc. Bullockia con especial énfasis en las especies afines al 'linaloe', B. aloexylon (Schiede ex Schlecht.) Engl.", a cargo de David Espinosa Organista. Los ejemplares recolectados presentan el mesosterno plano, por lo que pertenecen a la tribu Zygopini, de acuerdo con Lacordaire (1866); asimismo, tienen el segmento 2 del funículo antenal más largo que los otros segmentos, y los fémures carenados y armados con un diente ventral, caracteres que corresponden al género Macrocopturus Heller.

La historia taxonómica de Macrocopturus Heller está relacionada a Copturus Schönherr. El género Copturus, descrito por Schönherr en 1825, incluía la especie Poecilma papaveratum Germar, la cual fue designada por Bohemann como la especie tipo de Copturus. En 1895, Heller determinó que la mayoría de las especies de Copturus no se semejaban a C. papaveratus y designó un nuevo género (Zurus) para C. papaveratus y especies similares, y mantuvo cualquier otra especie en la concepción del género "Copturus". Heller describió varios subgéneros de "Copturus", incluyendo Macrocopturus. Desafortunadamente, la decisión de Heller estuvo en contra de las leyes de la nomenclatura, por lo que Wibmer y O'Brien (1986) decidieron que el nombre Copturus tenía que ser usado sólo para $C$. papaveratus y especies afines. Wibmer y O'Brien (1986) tomaron entonces el nombre Macrocopturus de Heller como nombre genérico de todas las especies que Heller incluyó en "Copturus", y designaron a Copturus satyrus Gyllenhal como la especie tipo. Basado en diferencias en la estructura del mesosterno, el verdadero género Copturus pertenece a la tribu Lechriopini y Macrocopturus a la tribu Zygopini. Macrocopturus Heller es un género americano con 146 especies (O'Brien y Wibmer 1982, Wibmer y O'Brien 1986), 48 registradas en Norte y Centroamérica y el Caribe, cinco de ellas con extensión a Sudamérica (O'Brien \& Wibmer 1982), y 98 especies distribuidas únicamente en Sudamérica (Wibmer \& O’Brien 1986). En México se presentan 18 especies, cuatro restringidas al territorio nacional (O'Brien \& Wibmer 1982).

Conforme al arreglo de Alonso-Zarazaga y Lyal (1999), en México se presentan 13 géneros de Zygopini. Superficialmente, los ejemplares de la especie desconocida tienen gran parecido a las especies del género Cylindrocopturus Heller; sin embargo, las especies de este grupo tienen los segmentos 1-2 del funículo antenal iguales en longitud y sus fémures carecen de dientes o carenas. Además de la forma del cuerpo, 
la especie nueva se distingue de los géneros Archocopturus, Copturomorpha, Helleriella, Hypoplagius, Phileas, Larides y Timorus porque éstos tienen los segmentos 1-2 del funículo casi de igual longitud (Heller 1895, Champion 1906). Asimismo, difiere de Peltophorus y Zygops porque éstos tienen el pigidio expuesto, y de Mnemynurus porque el rostro de éste es muy largo, casi llegando a la primera sutura ventral (Heller 1895, Champion 1906). En relación con los géneros Copturomimus y Hoplocopturus, que tienen también el segmento 2 del funículo antenal muy alargado, los ejemplares de la especie desconocida carecen de un área desnuda y estrigosa en el fémur anterior que caracteriza a las especies de Copturomimus (Champion 1906), y se distinguen de Hoplocopturus por el tamaño del rostro y forma del mesosterno, en este taxón el rostro es corto y el mesosterno tiene una depresión transversa limitada anteriormente por un margen arqueado (Heller 1895, Champion 1906).

Por lo anterior, los ejemplares de la especie desconocida se asignaron al género Macrocopturus. Las características de los especímenes no coinciden con ninguna de las especies reconocidas para este taxón, por lo que se consideran como miembros de una nueva especie. En este trabajo se describe esta especie, así como sus estados inmaduros.

\section{MATERIAL Y MÉTODOS}

Entre abril del 2004 y octubre del 2005 se capturaron nueve adultos, cinco pupas y 45 lavas. Cinco larvas fueron aclaradas con peróxido de hidrógeno (30 volúmenes) para observar las características morfológicas bajo el microscopio estereoscópico. Las medidas que se mencionan en la descripción de la nueva especie y de sus estados inmaduros fueron realizadas con un micrómetro ocular y se expresan en milímetros. Los tipos se encuentran depositados en la Colección Coleopterológica (FESZA) de la Facultad de Estudios Superiores Zaragoza, Universidad Nacional Autónoma de México, Ciudad de México.

\section{RESULTADOS \\ Macrocopturus burserophagus Muñiz \& Ordóñez, sp. nov. \\ Figs. 1-3}

\section{Holotipo macho}

Diagnosis. Esta especie se distingue de otras especies de Macrocopturus por tener el cuerpo proporcionalmente más ancho y plano en el dorso, así como por tener todos los fémures carenados y dentados.

Descripción: Cuerpo oval alargado; longitud 7.0, anchura 3.0; negro o marrón, antenas y tarsos marrón oscuro; vestidura densa de escamas mezcladas color blanco, pardo y marrón oscuro, excepto blancas alrededor de ojos, tercio basal de rostro, cuatro artejos basales de funículo antenal, escudete, sobre lados inferiores de 
Muñiz-Vélez† \& Ordóñez-Reséndiz: Especie nueva de Macrocopturus de Guerrero

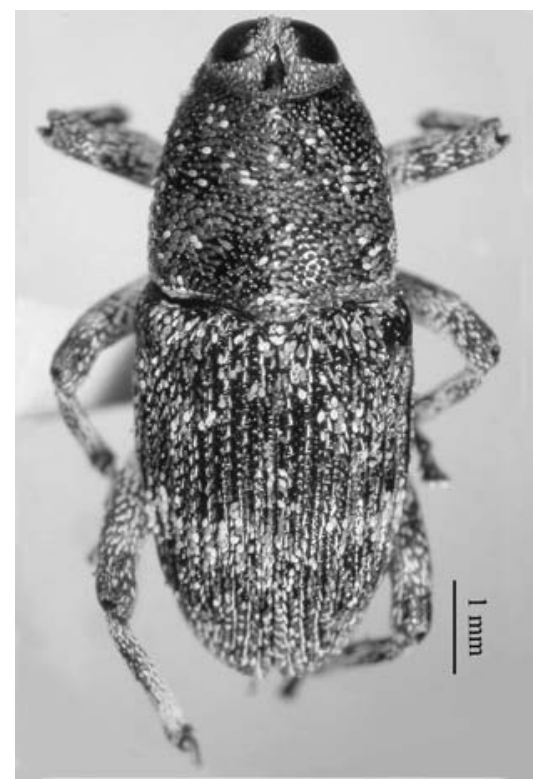

Figura 1. Vista dorsal de Macrocopturus burserophagus sp. nov.

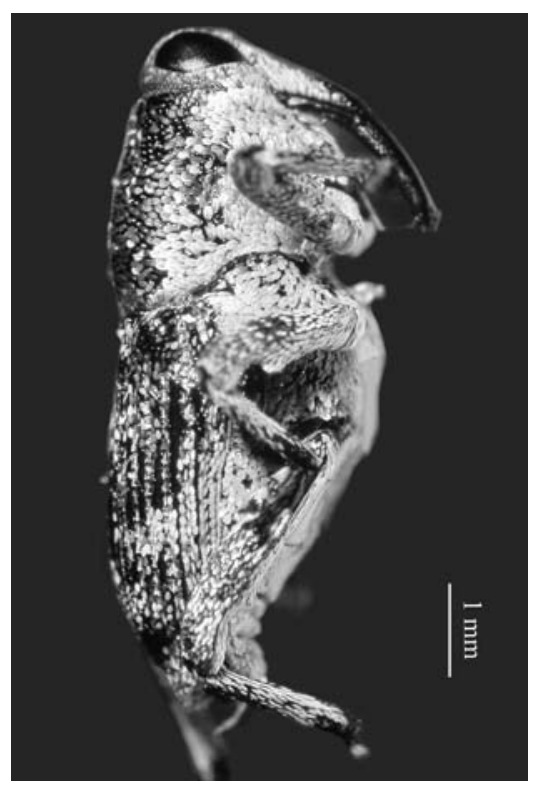

Figura 2. Vista lateral de Macrocopturus burserophagus sp. nov.

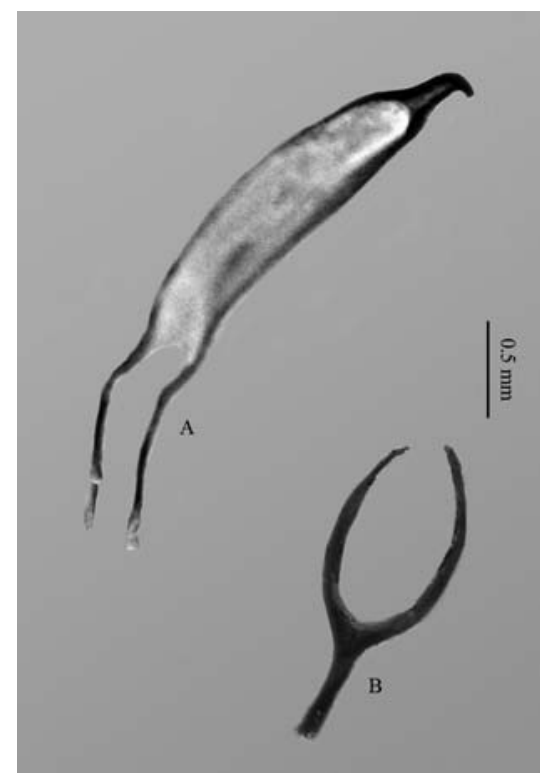

Figura 3. Genitalia masculina. A) Edéago, vista dorsal; B) Tegmen, vista dorsal. 
pronoto, meso-metasterno, coxas, patas y abdomen; escamas alargadas alrededor de ojos y patas, anchas y redondas en pronoto y élitros; patrón de escamas en élitros con tres bandas irregulares marrón oscuro: banda basal curva desde húmero a sutura elitral, banda media ancha ligeramente curva desde sutura a décima estría, banda posterior sobre callos apicales.

Cabeza redonda, pequeña; ojos muy grandes, lateralmente piriformes, rodeados por banda de escamas blancas, omatidias finas; vértex con área occipital triangular desnuda. Rostro delgado, longitud 2.24, ancho medio 0.69, casi tan largo como protórax, ligeramente curvo; superficie casi lisa, con punteado pequeño y sutil; quilla media poco marcada desde la base al inicio de las escrobas. Mandíbulas pequeñas y triangulares. Escrobas dirigidas hacia borde ventral. Antenas con escapo delgado; funículo con artejo 1 más corto que 2, 2 delgado y más largo que 3-4 juntos, 5-7 cortos, maza antenal oval alargada, acuminada, oscura, con suturas visibles. Pronoto 2.2 de largo, 2.7 de ancho; casi coniforme, borde basal sinuoso, borde apical algo cóncavo, lados casi rectos y ligeramente convergentes; superficie dorsal con punteado grueso en disco, puntos pequeños en ápice y borde basal; vista lateral con borde dorsal casi plano, borde basal diagonal hacia coxa protorácica, borde anterior sinuoso formando un débil lóbulo postocular. Escudete pequeño, ovalado. Élitros 4.2 de largo, 3.0 de ancho; húmeros poco marcados; estrías angostas, punteado cercano y rectangular; interestrías casi quilladas; declive elitral marcado por callos periapicales formados por interesarías 3-7; ápice elitral casi redondo. Vista ventral: prosterno excavado hasta borde anterior de coxas mesotorácicas, poco profundo, bordes laterales quillados; coxas protorácicas separadas por espacio casi igual al ápice rostral; mesosterno plano, en declive; mesepímero y mesepisterno trianguliformes; metasterno casi convexo, borde redondeado, con surco delgado que no alcanza borde anterior; metaepisterno rectangular, casi desnudo. Abdomen en vista lateral, ventritos 1-4 ascendentes, 5 horizontal; ventrito 1 casi tan largo como 2-3 juntos, poco ahondado en parte central; ventrito 2 casi rectangular, más largo que 3-4 unidos, ángulos lateroposteriores proyectados hasta sutura posterior de esternito 3; ventritos 3-4 cortos, rectangulares, casi quillados en parte media; ventrito 5 trapezoidal, lados gruesos y biselados, borde apical algo cóncavo. Patas delgadas y relativamente largas; fémures unidentados, más o menos lineales, base casi tan ancha como ápice, quilla en parte media de cara externa; fémures metatorácicos alcanzan ápice de abdomen; tibias casi lineales y ungiculadas; tarsos $1 / 2$ de largo que tibia; tarsito 1 más corto que 2-3 combinados; tarsito 3 bilobulado, más ancho que 2; tarsito 5 casi tres cuartas partes de largo que 1; uñas simples, separadas y delgadas. Genitalia: edéago 1.85 de longitud y 0.42 de anchura máxima, cilíndrico, ápice en ángulo de 90 grados; vista dorsal con lados paralelos y convergentes hacia ápice; saco interno con canal eyaculador visible; apodemas 0.8 de largo, filiformes, extremo basal poco 
ensanchado. Tegmen en forma de horquilla, longitud 1.24, con piezas basales unidas al manubrio en línea media ventral y separadas en línea media dorsal.

Variación intraespecífica (paratipos): Longitud 6.9-7.4, ancho 2.9-3.45. Valores promedio: pronoto 2.38 de largo, 2.84 de ancho; élitros 4.27 de largo, 2.98 de ancho.

Serie tipo: Holotipo macho y ocho paratipos (siete machos y una hembra), etiquetados con los siguientes datos: holotipo "México:Guerrero, Cerro Alto El Tepehuaje, $17^{\circ} 6^{\prime} 29^{\prime}$ N, $99^{\circ} 29^{\prime} 18.7$ 'W, 700m, SBC, 20-V-2004, D.Espinosa, col.”; tres machos "México:Guerrero, Salto Valadez, 17²7'42.98"N, 99²7'32"W, 1400m, SBC, 25-VI-2004, D.Espinosa, col."; cuatro machos y una hembra "México:Guerrero, Cerro Alto El Tepehuaje, 176'29”N, 99²9'18.7”'W, 700m, SBC, 20-V-2005, D.Espinosa, col.".

Etimología: Hace alusión al género del vegetal donde se encuentra y se desarrolla el gorgojo, la especie Bursera citronella Mc Vaugh \& Rzedowski.

Hábitat: En ramas jóvenes de B. citronella que crece en manchones de selva baja caducifolia, aislada en peñas de rocas cristalinas del Cretácico (mármol). Clima Aw"2ig, cálido subhúmedo con lluvias de verano, sequía intraestival y régimen de temperaturas isotermal tipo Ganges (temperatura máxima antes del solsticio de verano). El insecto pupa dentro de la rama cuaternaria de B. citronella.

Distribución geográfica: México, Guerrero: Cerro Alto El Tepehuaje, Municipio Juan R. Escudero y Salto Valadez, Municipio Chilpancingo de los Bravos.

\section{Larva}

Figs. 4-5

Descripción: color blanco-amarillento, cápsula cefálica y dorso del primer segmento torácico ambarino, mandíbulas negras. Longitud 6.5-10, ancho máximo 1.5-2.5. Cabeza retraída en primer segmento torácico, más angosta que pronoto; longitud 1.37-1.85, ancho máximo 0.7-1. Forma oval alargada. Tegumento en su mayor parte liso, color ámbar, con banda longitudinal más clara a ambos lados de línea media, región apical de la frente color ámbar oscuro. Sutura epicraneal alargada, sutura frontal visible. Frente y área epicraneal con sedas cortas y largas. Una seda frontal alargada a cada lado, próxima a unión con clípeo, ocho sedas anteriores agrupadas en grupos de cuatro; 16 sedas epicraneales variables, alineadas a lo largo de sutura frontal a ambos lados. Clípeo membranoso, trapezoidal, con dos pares de sedas medianas inferiores. Labro semicircular, esclerosado, ambarino, con dos sedas centrales alargadas, una seda lateral externa alargada y dos sedas medianas anteriores. Mandíbulas robustas, cónicas, ligeramente convexas, tegumento rugoso, base con dos sedas medianas. Maxilas grandes, ambarinas. Estipe ligeramente más ancho en ápice, margen interno sinuoso. Dos sedas en la base del palpo y una en primer tercio basal, más larga. Palpo maxilar de dos segmentos, el primero ancho y truncado con la mitad anterior pigmentada, el segundo más estrecho, alargado e 
igualmente pigmentado. Labio con prementón grande y ancho, una seda lateral a cada lado; postmentón grande con par de sedas laterales, una posterior alargada y una anterior algo más corta. Palpo labial de dos artejos, el basal ancho y el apical más estrecho, cónico y rosado. Antenas de dos segmentos, el primero membranoso, casi triangular, con dos sedas dorsolaterales externas cortas, segmento distal corto y angulado. Tórax. Protórax con la parte dorsal esclerosada con dos áreas bien definidas y divididas por una elevación posterior. Anteriormente con seis sedas marginales superiores, alargadas y fusiformes, dos centrales cortas aciculares. Dos sedas protorácicas laterales alargadas cerca de elevación posterior. Área posterior convexa y oblicua, con microsensilas y una seda corta a ambos lados de línea media y una seda alargada en cada extremo. Estigma lateral alargado y ovalado con una seda dorsal larga, epipleura trapezoidal provista de dos sedas alargadas, pleura triangular con seda media alargada, lóbulo pédico con ocho sedas variables, dos anteriores, cinco medias y una inferior. Esterno con dos sedas marginales medianas alargadas, mesotórax con dos pliegues cubiertos de microsensilas.

Abdomen de diez segmentos, presentando los primeros siete, prescudo, escudo y estudete. Prescudo con dos sedas medianas alargadas, escudo con tres sedas alineadas a cada lado, una más alargada. Octavo segmento con una seda a cada lado.

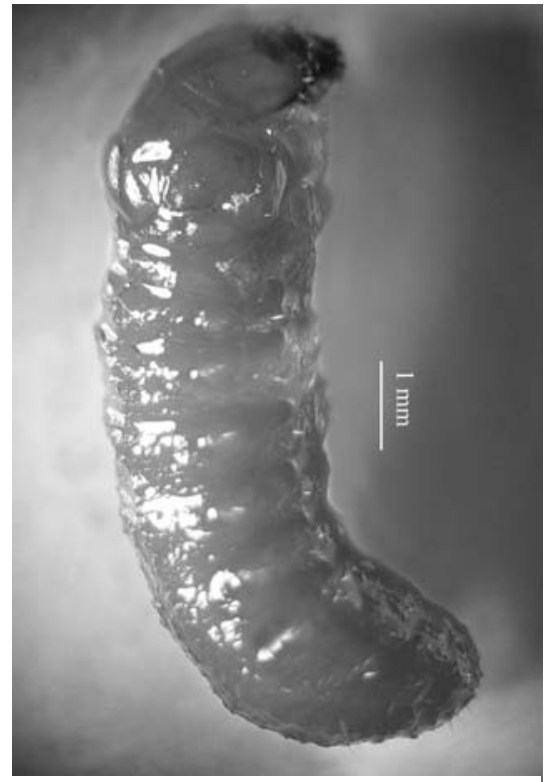

Figura 4. Vista lateral de la larva de Macrocopturus burserophagus sp. nov.

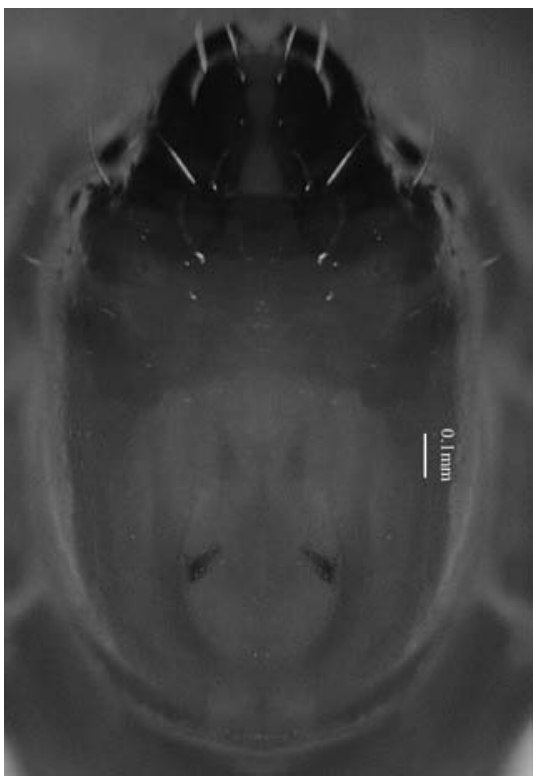

Figura 5. Cápsula cefálica. 


\section{Pupa}

Figs. 6-7

Descripción: Exarada, cuerpo cilíndrico, color amarillo. Longitud 9.5-10.5, diámetro 2.5-3 en su porción más ancha, a la altura de patas metatorácicas. Cabeza bien definida; canto ocular marcado, grande, ocupa 2/3 partes de cabeza. Rostro moderadamente largo, tan largo como pronoto, reposa entre coxas protorácicas más allá de coxas mesotorácicas; con seda pequeña a cada lado, ubicada entre inserción antenal y mandíbulas. Mandíbulas esclerosadas, pequeñas y dentadas. Antenas acodadas, paralelas al rostro; escapo pequeño, no alcanza borde anterior de pronoto; funículo más largo que escapo, segmento 2 más largo que 1; maza bien desarrollada, con segmentación visible. Pronoto coniforme, convexo, base ancha, borde anterior ligeramente estrecho. Superficie dorsal con 12 sedas pequeñas, tres pares alineados cerca de línea media y un par en ángulo antero-lateral. Mesotórax trapezoidal, con dos pares de sedas centrales pequeñas; escudete bien definido, con un par de sedas pequeñas. Metatórax rectangular, con dos pares de sedas pequeñas,

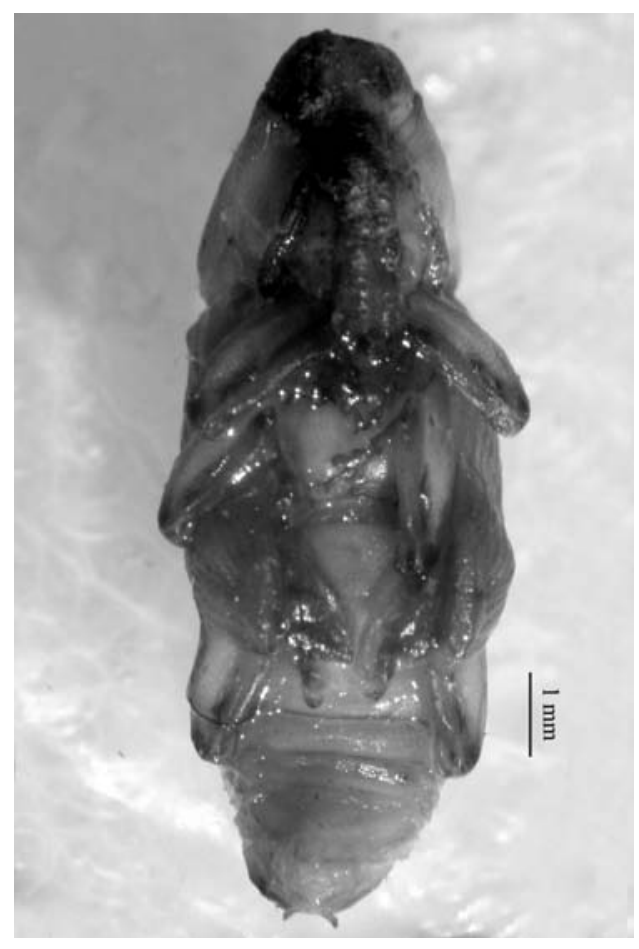

Figura 6. Vista ventral de la pupa de Macrocopturus burserophagus sp. nov.

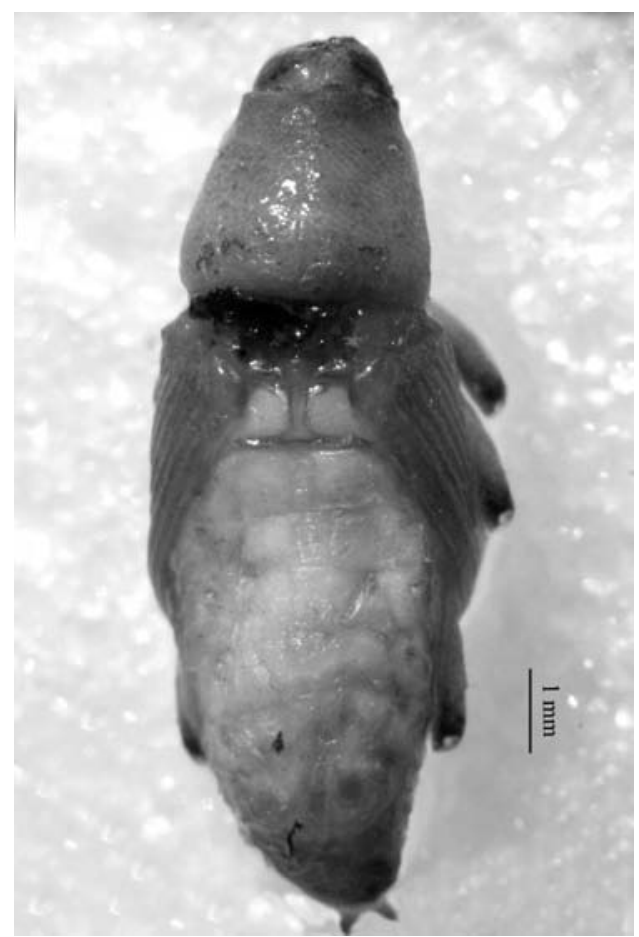

Figura 7. Vista dorsal de la pupa de Macrocopturus burserophagus sp. nov. 
un par central y otro en borde de alas. Abdomen cilíndrico y glabro. Vista dorsal con nueve segmentos bien definidos de tamaño decreciente; segmento 1 rectangular y convexo, casi del mismo ancho que metatórax; segmento 9 trapezoidal, con los urogomphi; cada segmento con dos sedas en dorso y una en lóbulo pleural. Vista ventral con cuatro segmentos bien definidos, esternito 1 muy convexo, más grande que 3-4 juntos. Apéndices. Élitros con estrías e interesarías marcadas, con escamas y punteado más o menos definido; en lado ventral alcanzan el fémur metatorácico y casi la mitad del primer segmento abdominal. Alas metatorácicas más largas que élitros. Patas bien desarrolladas. Fémures dentados y con una seda pequeña cerca de tibia. Tibias unguiculadas. Tarsos criptopentámeros. Uñas simples, divergentes.

\section{DISCUSIÓN}

Como se mencionó en la introducción, a simple vista, Macrocopturus bruserophagus se parece más a las especies mexicanas de Cylindrocopturus que a cualquier especie descrita de Macrocopturus. Formas típicas de este género, como las especies $M$. cincticollis, M. maculosus y M. undatus, descritas por Champion (1906) para Panamá, se semejan un poco a $M$. bruserophagus, pero son proporcionalmente más estrechas y menos aplanadas dorsalmente. M. bruserophagus concuerda con $M$. maculosus en el largo del segundo artejo del funículo antenal, que es tan largo como el tercero y cuarto juntos (Champion 1906). Al igual que M. cincticollis y M. undatus presenta estrías e interestrías casi del mismo ancho. El punteado del protórax y la carena media lo semejan a M. maculosus y M. undatus (Champion 1906), pero en éstos la carena es débil y no completa como en M. bruserophagus. Difiere de todas las especies del grupo I de Champion (1906) por el carácter de los fémures, en $M$. bruserophagus todos están carenados en la parte media de la cara externa, en cambio las otras especies presentan uno o dos fémures carenados en la cara externa, que pueden ser meso o metatorácicos. En cuanto al patrón de coloración de la vestidura, la de $M$. burserophagus es jaspeada, blanca con negro, muy diferente a la que presentan las demás especies.

AGRADECIMIENTOS. La segunda autora agradece a David Espinosa Organista por los ejemplares donados (proyecto BS001 de CONABIO). Enrique Morelli, Universidad de la República (Uruguay), efectuó valiosas correcciones a la descripción de la larva. Ricardo Baltazar Covarrubias realizó parte del registro fotográfico y proporcionó asesoría técnica. Juan J. Morrone y cuatro revisores anónimos hicieron aportaciones importantes para la conclusión de este trabajo. La Carrera de Biología de la Facultad de Estudios Superiores Zaragoza (UNAM) contribuyó con diversos apoyos. 


\section{LITERATURA CITADA}

Alonso Zarazaga, M.A. \& C.H.C. Lyal. 1999. A world catalogue of families and genera of Curculionoidea (Insecta: Coleoptera) (excepting Scolytidae and Platypodidae). Entomopraxis, Barcelona, $315 \mathrm{p}$.

Champion, G.C. 1906. Insecta. Coleoptera. Rhynchophora. Curculionidae. Curculioninae. Pp. 69-87. In: Champion, G.C. 1906-1909. Biologia Centrali-Americana. Vol. IV, Part 5. i-viii + 513 pp.

Heller, K.M. 1895. Zygopiden-Studien II, mit besonderer Berücksichtigung der gattung Copturus. Abhandlungen und Berichte des Koeniglichen Zoologischen und Anthropologisch-Ethnographischen Museums zu Dresden (Abh. Ber. Mus. Dres.), 1894/95, (11):1-70.

Hespenheide, H.A. 2002. VIII. Conoderinae Schoenherr 1833. Pp. 754-756. In: Arnett, R.H. Jr., M.C. Tomas, P.E. Skelley \& J.H. Frank (Eds.). American Beetles. Polyphaga: Scarabaeoidea through Curculionoidea. Volume 2. CRC Press LLC. Boca Raton, Florida.

Kojima, H. \& Ch. H. Lyal. 2002. New Oriental and Australian Conoderinae, with Taxonomic Notes on the Tribe Othippiini (Coleoptera: Curculionidae). ESAKIA, 42:161-174.

Lacordaire, T. 1866. Histoire naturelle des Insectes. Genera des Coléopteres on exposé méthodique et critique de tous les genres proposés Jusqu'ici dans cet ordre d'insectes. Vol. 7. Paris, Roret. 620 pp.

O'Brien, C.W. \& G.J. Wibmer. 1982. Annotated checklist of the weevils (Curculionidae sensu lato) of North America, Central America, and the West Indies (Coleoptera: Curculionoidea). Memoirs of the American Entomological Institute, 34: 1-381.

Schönherr, C.J. 1825. Curculionides [Tabula synoptica familiae curculionidum]. Isis von Oken, 5:c.581-588.

Wibmer, G.J. \& C.W. O’Brien. 1986. Annotated checklist of the weevils (Curculionidae sensu lato) of South America (Coleoptera: Curculionoidea). Memoirs of the American Entomological Institute, 39:12-14, 17, 20, 272-274. 